\title{
A OBSERVATIONAL STUDY OF ENDOSCOPIC REPAIR OF CSF RHINORRHOEA
}

Dr. Bijoy Krishna Dept. of ENT; Institute of Otolaryngology and Head \&Neck Surgery; IPGMER; Kolkata; Bhadra India.

\begin{tabular}{ll}
\hline $\begin{array}{l}\text { Dr. Soumen } \\
\text { Biswas* }\end{array}$ & $\begin{array}{l}\text { Dept. of ENT; Institute of Otolaryngology and Head \&Neck Surgery; IPGMER; Kolkata; } \\
\text { India. *Corresponding Author }\end{array}$ \\
\hline Dr. Debarshi Jana & $\begin{array}{l}\text { Institute of Post-Graduate Medical Education and Research, A.J.C. Bose Road, Kolkata, } \\
\text { West Bengal, India-700020. }\end{array}$ \\
\hline
\end{tabular}

\section{ABSTRACT}

Background: Intrathecal fluorescein may be used to visualize CSF fistulas using a nasal endoscope. This allows the examiner to directly visualize the size of the defect, its location, and its rate of flow. The first repair of CSF leak was performed by Dandy in 1926 using a frontal craniotomy. This technique had a $60-80 \%$ success rate and was the gold standard for decades. In 1964Vrabec and Hallberg described the endonasal approach of CSF leak repair.

Aims: This study aims to establish the success rate of endoscopic surgical repair of CSF rhinorrhoca.

Materials And Methods: The study was conducted in Department of ENT IPGMER and SSKM Hospital. During the study period of 18 months, 30 patients have been admitted in our department with CSF leak (most of them were referred from neurosurgery).

Results: Fat and fascia lata and temporalis fascia were used to repair CSF leak in 24 patients. Bath plug technique was applied for 3 patients. Repair with vascularised flap such as Hadad flap or middle turbinate graft were used in case of 3 patients. CSF rhinorrhoea repair was successful in 28 out of 30(94\%) patients after primary surgery whereas recurrence occurred in 2(6\%) cases. Among them 1 patient underwent second surgery which achieved definitive closure of the leak. But one case lost follow up and did not come for second surgery. Interval between surgery and recurrence was varied and so were the causes.

Conclusion: CSF rhinorrhoea cases can be repaired by an endoscopic approach with a high success rate. Radiological investigation of PNS and brain are equally important for selection of cases for endoscopic repair. Spontaneous leak must be checked cautiously to exclude features of raised ICT. Relapse occurs mainly for failure to delineate actual site or sites of leak and inadequate size or faulty placement of graft. Success depends not only on surgical skill but also on baseline intracranial tension, cause \& site of leak and postoperative care.

\section{KEYWORDS}

\section{Endoscopic Repair, CSF, Rhinorrhoea}

\section{INTRODUCTION}

Spontaneous CSF leaks are difficult to diagnose. CSF leak was first described by Galen in 200B.C. Saintclair Thompson reported the first series of leaks in 1889. Most of the times patient presented to the outpatient department with unilateral rhinorrhoea, headache, recurrent meningitis.

Thorough histories followed by bio-chemical and radiological investigations are necessary for diagnosing CSF rhinorrhoea. When performing the history, it is important to ask about onset, duration, severity, laterality, and associated symptoms. History of facial trauma, sinus surgery or neurosurgical procedures should be noted. Physical examination requires a complete otorhinolaryngological examination including cranial nerve testing, nasal endoscopy, nasal endoscopy with intrathecal fluorescein. Findings include bony deformity, visualization of defect, and clear rhinorrhea. Differential diagnosis includes allergic rhinitis and autonomic dysfunction.

Biochemical testing begins with analysis of the nasal secretions ${ }^{1}$. Beta2 -transferrin is a protein found in CSF. perilymph, and aqueous humor. It is produced by neuraminidase activity in the brain. Once fluid is collected, an electrophoresis is performed to detect the protein. This is a highly sensitive and specific assay that has been used for the detection of CSF in fluids. The only drawback to beta-2-transferrin is the time it takes for analysis. A newer assay that detects beta- trace protein has been developed and only takes fifteen minutes to perform. Beta trace protein is the second most abundant protein found in CSF. Its concentration in CSF is thirty-five times more than in serum. The assay has a similar sensitivity and specificity to the beta-2-transferrin assay, but it is contraindicated in diseases that increase serum beta trace or decrease CSF beta traces such as meningitis and renal failure. In addition to these screening tests, imaging must be performed to identify possible locations of dural defects.

Radiological imaging includes high resolution CT, magnetic resonance imaging, radioisotope cisternography, metrizamide CT cisternography, and MR cisternography.

CT provides information about bony detail, bone defects, and can help to plan surgical approaches. The posterior table of the frontal sinus and the lateral and posterior walls of the sphenoid are best assessed using the axial plane, while the cribriform plate, fovea, and sphenoid roof are best visualized in the coronal plane. When clinically indicated, like as in RTA HRCT of the temporal bone is also indicated.

MRI can be used when an encephalocele is the cause of CSF rhinorrhea. MRI can elucidate the contents of meningoceles, which can contain large vessels, or actual brain matter. MR cistemography is a recently developed technique that uses a fast spin-echo sequence with fat suppression and image reversal to highlight CSF fistulas. This technique may supplant other forms of cisternography that require intrathecal administration of contrast material such as metrizamide CT cisternography, or radioisotope cisternography.

Intrathecal fluorescein may be used to visualize CSF fistulas using a nasal endoscope. This allows the examiner to directly visualize the size of the defect, its location, and its rate of flow. The first repair of CSF leak was performed by Dandy in 1926 using a frontal craniotomy. This technique had a $60-80 \%$ success rate and was the gold standard for decades. In 1964Vrabec and Hallberg described the endonasal approach of CSF leak repair. In 1981, the first endoscopic CSF rhinorrhea repair was performed by Wigand ${ }^{2}$. This has become the standard of care due to less morbidity and $90 \%$ first time success rate. The key to endoscopic surgical repair of CSF rhinorrhea is an accurate preoperative and per-operative assessment of location of the fistula, its dimensions, and the anatomy of the surrounding area. Sites of lesions include cribriform plate, ethmoid, sphenoid, and frontal sinuses most of which are easily assessable transnasally. Post-operative follow-up for 6 months is mandatory in all these patients. All the patients should be evaluated with diagnostic nasal endoscopy. After successful repair of CSF leak recurrence can happen particularly in high pressure leak which should be treated accordingly.

This study aims to establish the success rate of endoscopic surgical repair of CSF rhinorrhoca which depends on accurate preoperative and per-operative assessment of location of the fistula, its dimensions, and the anatomy of the surrounding area. Sites of lesions include cribriform plate of ethmoid and sphenoid sinus most of which are easily accessible transnasally. 
MATERIALS AND METHODS:

\section{Place Of Study:}

This was an observational longitudinal study. The study was conducted in Department of ENT of IPGMER and SSKM \& Hospital.

\section{Period Of Study:}

The study was conducted for a period of 18 months (November 2017 September 2019).

\section{Study Population:}

During the study period of 18 months, 30 patients have been admitted in our department with CSF leak (most of them were referred from neurosurgery).

\section{Inclusion Criteria:}

1. Only the patient having spontaneous and traumatic CSF leak were included in our study

2. Patients having CSF leak due to iatrogenic trauma especially following FESS were taken.

3. Patients within the age group of 18-60 years were selected for study.

\section{Exclusion Criteria:}

1) Patients tuning CSF leak due to intra-cranial space occupying lesion were excluded from our study.

2) Patients below the age of 18 years and above the age of 60 years were excluded from our study.

\section{RESULT AND DISCUSSION}

Cerebrospinal fluid (CS) leaks are generally classified as spontaneous idiopathic, traumatic, jatrogenic ${ }^{3}$. Traumatic causes include both blunt and penetrating facial injuries. Iatrogenic causes include neurosurgical and otolaryngologic approaches to neoplastic disease, as well as functional endoscopic sinus surgery (FESS). Most spontaneous or primary causes of CSF rhinorrhoea are now thought actually to be secondary to elevations in intracranial pressure (ICP) that might be seen in patients with idiopathic intracranial hypertension $(\mathrm{IIH})^{5}$ Congenital skull base defects can also lead to CSF rhinorrhea.

In our study $25(83 \%)$ of CSF rhinorrhoea was caused by trauma ( 5 surgical trauma 20 non-surgical trauma). $17 \%$ of our cases presented with spontaneous CSF leak. Presence of spontaneous CSF leak was variable in literature, ranging from $4-39 \%{ }^{4}$. Wax et al. ${ }^{6}$ showed that the incidence of spontaneous CSF leakage was $29.5 \%$. The majority of patients with a CSF leak due to accidental trauma (eg, motor vehicle accident) present immediately. Most of the patients (95\%) with a delayed CSF leak present within 3 months after the injury. In contrast to traumatic leaks, only $50 \%$ of patients with iatrogenic CSF leaks present within the first week after the insult. In most cases, the patient will have been discharged when the leak presents itself.

Hence, educating the patient regarding the common symptoms associated with a CSF leak such as salty or metallic taste is of paramount importance. Any surgical manipulation near the skull base can result in an iatrogenic CSF leak? Skull base injuries can vary from simple cracks in the bony architecture to large $\mathrm{p} 1 \mathrm{em}$ ) defects with disruption of the dura and potentially brain parenchyma

Otolaryngology procedures, including FESS can lead to a skull base defect and CSF rhinorrhea. Certain neurosurgical procedures such as craniotomy and transsphenoidal pituitary resections are most commonly associated with an increased risk of CSF rhinorrhea.

Defects in the closure of the anterior neuropore can result in the hemiation of central nervous tissue through anterior cranial fossa. These are infrequently associated with CSF rhinorrhea.

The embryologic efect is typically a patent fonticulus frontalis or foramen cecum.

Meningoencephaloceles usually present in childhood as an intranasal/extranasal mass that transilluminates and expands with crying (Furstenberg sign). A high index of suspicion should be maintained with all pediatric intranasal masses, particularly those occurring at the midline. A biopsy should never be obtained unless a complete imaging workup has been conducted.

This terminology seems to imply that spontaneous CSF leaks are idiopathic in nature, however, recent evidence has led us to realize that spontaneous CSF rhinorrhea may in reality be secondary to an elevated intracranial pressure (ICP).

Despite the multifactorial causes of elevated ICP, once this problem ensues, the pressure exerted on areas of the anterior skull base such as the lateral lamella of the cribriform or lateral recess of the sphenoid sinus results in bone remodeling and thinning. Ultimately, a defect is formed. At This point, the dura herniates through the defect (meningocele). If the defect is large, brain parenchyma may also herniate through the defect (encephalocele)

Anterior cranial fossa is most vulnerable to head trauma and iatrogenic injuries. The most affected site of CSF leak was lateral lamella of the cribriform plate where bone is thinner and dura's adherence to the bone is stronger ${ }^{8}$. In our study we got $87 \%$ leak in cribriform plate $13 \%$ leak in sphenoid sinus.

A thorough history is the first step toward accurate diagnosis. The typical history of a cerebrospinal fluid (CSF) leak is that of clear, watery discharge, usually unilateral. Diagnosis is made more easily in patients with recent trauma or surgery than in others. Delayed fistulas are difficult to diagnose and can occur years after the trauma or operation. These cases often lead to 1 misdiagnosis of allergic and vasomotor rhinitis. On occasion, the patient has a history of headache relieved by drainage of CSF. Drainage may be intermittent as the fluid accumulates in one of the paranasal sinuses and drains externally with changes in head position (ie, reservoir sign).

A history of headache and visual disturbances suggests increased intracranial pressure. Sometimes, associated symptoms can assist in localizing the leak. For example, anosmia (present in $60 \%$ of individuals with post-traumatic rhinorrhea), indicates an injury in the olfactory arca and anterior fossa, especially when it is unilateral. Optic nerve deficits suggest a lesion in the region of tuberculum sellae, sphenoid sinus, or posterior ethmoid cells. Patients with recurrent meningitis, especially pneumococcal meningitis, should be evaluated for a defect that exposes the intracranial space to the upper airway, regardless of the presence or absence of CSF rhinorrhea.

Physical examination should include complete rhinologie (including endoscopic), otologie, head und neck, and neurologic evaluations. Drainage of CSF in some cases may often be elicited on endoscopy by having the patient perform a Valsalva maneuver or by compressing both jugular veins (Queckenstedt-Stookey test). However, most of the time physical examination is unrevealing, especially in patients with intermittent CSF rhinorrhea.

In patients with head trauma, a mixture of blood and CSF may make the diagnosis difficult CSF separates from blood when it is placed on filter paper, and it produces a clinically detectable sign: the ring sign, double-ring sign, or halo sign. However, the presence of a ring sign is not exclusive to CSF and can lead to false-positive results. In contras to unilateral rhinorrhea, bilateral rhinorrhea gives no clue of the laterality of the defect. However, even in this situation, exceptions can occur. Paradoxical rhinorrhea occurs when midline structures that act as separating barriers (eg, crista galli, vomer) are dislocated. This dislocation allows CSF to flow to the opposite side and manifest at the contralateral naris

Only the patient having spontaneous and traumatic CSF leak were included in our study patients having CSF leak due to iatrogenic trauma especially following FESS were taken. Patients having CSF leak due to intracranial space occupying lesions were excluded from our study. Site of the the leak was confirmed by CT cisternography and diagnostic nasal endoscopy (DNE). The CT cisternography evaluation after the intrathecal administration of low-osmolar, nonionic iodine Contrast media (e.g., metrizamide), revealed precise sites of leak in 28 cases. The primary problem with CT cisternography is that identification of the exact location of the leak requires the presence of an active fistula. Intermittent leaks that are temporarily sealed by swelling, inflammation, or prolapsed arachnoid may yield a falsenegative result

In contrast, magnetic resonancecisternography does not require the intrathecal administration of a contrast agent. On 12 weighted MRI cerebrospinal fluid gives a very high signal while bone gives a low signal. The resulting high contrast may enable clear visualization of the site of defect. The criteria that include localization of fistula are (1) demonstration of brain hernia through cribriform plate or paranasal 
sinus (2) demonstration of high signal through cribriform plate or paranasal sinus, the signal being continuous and similar to that in basal cisterns ${ }^{9}$.

Combining these two tests may give the highest sensitivity and specificity but is not cost effective.

Overall male to female ratio in this study was 1:4 which confirm the previous observation that Women affected four times more frequently than men. ${ }^{10}$

The age of the patient in this study population was $18-60$ years. Almost $50 \%$ patients were belonged to age group of $31-40$ years supporting the commonest occurrence in middle aged adults.

It was four times more common in urban people in comparison to rural people. Probably it reflects increased number of road traffic accidents in urban areas. Study of occupation revealed that outgoing people are more susceptible to get this type of leak.

In our study we found cribriform plate was the commonest site for CSF leak. Anterior cranial fossa is extremely vulnerable to both head trauma and iatrogenic injuries. Because dura in this the region is tightly adherent to bone.

Treatment of CSF rhinorrhoea may be conservative or surgical. But conservative treatment resolves the defect only in $50 \%$ of the cases and cannot exclude risk of mening it is Key the success of surgical repair is to correctly locate the site of leak. Hyperventilation done by anaesthetists during surgery proved to be quite effective in raising the ICT thereby causing gush of CSF leak and thus confirming the actual leak site during surgery. Presutti et $\mathrm{al}^{11}$ has reported good result with intraoperative increase in intracranial pressure by this method. Though fat and fascia lata suffice to seal small leaks newer techniques like nasoseptal neurovascular pedicled flap as well as free flaps proved to be superior regarding rapid healing and lesser chance of displacement. Experience of different authors varies regarding graft material. Selection of material and technique should be individualized according to site, size and cause of leak. Though fat and fascialata suffice to seal small leaks newer techniques like nasoseptal neurovascular pedicle flap as well as free flaps proved to be superior regarding rapid healing and lesser chance of displacement. ${ }^{12}$

For small to medium size leak fat and fascia lata harvested from thigh has been used in 24 cases). For larger leak neurovascular pedicle flap has been usedímucoperiosteal flap from middle turbinate in Icase, Hadad's flap from septal mucoperichondrium in 2 cases). Only fat was used in case of very small leaks in 3 patients. In all cases grafts were secured in place by gelfoam followed by merocel packing. All graft materials were placed in overlay technique. Use of the lumbar drain is controversial[ 5.3] in the post-operative period. We did not use lumbar drain for any patient. Intracranial pressure was well controlled with diuretics, Success rate (93\%) of this case series is comparable with that found in literature i.e. $80-100 \%$ and $7 \%$ recurrence rate for endoscopic approach. Failure rate with intracranial approach is rather higher (20$40 \%$ ). In this case series all two cases of recurrence belonged to the spontaneous group and one among them had features of raised ICT in $\mathrm{CT}$ scan and MRI brain. In one case leak recurred in a new site. This is because the leak acts as a pressure valve in patients with persistently elevated ICP. As soon as it is surgically closed, leak can reappear in any of the thinnest areas of the skull base (cribriform plate or walls of the highly pneumatized paranasal sinuses). This current theory is supported by evidence from studies by Schlosser ${ }^{13}$ that found symptoms suggestive of elevated ICP (pulsating tinnitus and headache in successfully closed fistulae), indicating that the intervention might have restored the preoperative hypertensive state.

It has been found that patients with spontaneous CSF leak with high intracranial tension have got surgical failure. Undiagnosed cases of high pressure higher chance $(46 \%)$ of hydrocephalus with past history of cerebrovascular accident, trauma, subarachnoid haemorrhageetc have a high chance of recurrence. These patients must be counselled properly and additional intervention may be required like lumber drain even VP shunting. Thus it is of utmost importance to look for clinical and radiological features suggestive of raised intracranial tension before surgical planning.

Considering lower mortality, cost, hospital stay and higher or similar success rate as compared to intracranial approach, endoscopic repair is becoming the procedure of choice for repair of CSF rhinorrhoea

\section{CONCLUSION}

The following were the findings of our study in a nutshell:

The gender distribution revealed that there was a preponderance of female patients with a

1) Male-female ratio of 1:4. The most common occurrence of CSF leak was in the fourth decade.

2) Study of occupation revealed that working people are more susceptible to CSF leak.

3 ) In our study the most common causative factor is trauma (66.7\%).

4) The most common comorbid factors associated are cough and constipation.

5) In our study unilateral watery discharge from the nose is the predominant symptom.

6) In our study the commonest site of leak is medial lamella(50\%).

7) In our study $83 \%$ of patients presented with spontaneous CSF leak

8) In operative approach we had to do septoplasty in $40 \%$ of patients to create space.

Way in our study fat and fascia lata and temporalis fascia was used to repair CSF leak in 24 patients. Bath plug technique was applied for 3 patients. Repair with vascularised flaps such as Hadad flap or middle turbinate graft were used in case of 3 patients.

1) In this case series all two cases presented with recurrence. They belonged to the spontaneous group and one among them had features of raised ICT in CT scan and MRI brain. In one case a leak recurred in a new site.

2) In our study chronic headache and anosmia are common postoperative complications.

\section{REFERENCES}

1. Schnabel $\mathrm{C}$, et al. Comparison of B2-transferrin and trace protein for detection of Cerebrospinal Fluid in Nasal and Ear Fluids Clinical Chemistry 2004;50-661-663. Wigand ME Transnasalethmoidectomy under endoscopic control. Rhinology 1981:19-7. 2. Wigand ME Transnasalethmoidectomy under endoscopic control. Rhinology 1981:19-7.
3. 26. P Paul, Kiran Upadhyay, Indian Journal of Neurotrauma (INT).2010, Vol. 7, No. 1, $67-70$

4. Badia L. Loughran S, Lund V. Primary spontaneous cerebrospinal fluid rhinorrhea and obesity. Am J Rhinol. 2001;15:117-119.

5. Andrey S. Endonasal Endoscopic Repair of Spontaneous Cerebrospinal Fluid Leaks. Arch otolaryngol head neck surg/vol 129, aug 2003:859-863.

6. Wax MK, Ramadan $\mathrm{HH}$, Ortiz $\mathrm{O}$, et al. Contemporary management of cerebrospinal fluid rhinorrhea. Otolaryngol Head Neck Surg. 1997; 116:442-449.

7. Kelley TF, Stankiewicz JA, Chow JM, Origitano TC, Shea J. Endoscopic closure of postsurgical anterior cranial fossa cerebrospinal fluid leaks. Neurosurgery 1996,39.7434

8. Yessenow RS McCabe BF. The osteomucoperiosteal flap in repair of cerebrospinal fluid rhinorrhea:a 20-year experience Otolarygol Head Neck Surg 1989;101:555-8.

9. CalcaterraTC. Diagnosis and management of ethmoid cerebrospinal thinorrhea OtolaryngolClin North Am 1984;18(1):99-105.

10. Lanza DC, O'Brien DA, Kennedy DW. Endoscopic repair of cerebrospinal fluid fistulae andencephaloceles. Laryngoscope. 1996;106:1119-1125.

11. Presutti L, Mattioli F, Villari D, Marchioni D, Alicandri-Ciufelli M Transnasa treatment of cerebrospinal fluid Otorhinolaryngologica Italica. 2009 Aug;29(4):191

12. Hadad G, Bassaquastequy, Cassau RL, Mataza JC, Kassam A, Synderman CH, Mintz A.A novel reconstructive technique after endoscopic expanded endonasal approaches: vascular pediclednasoseptalflap.Laryngoscope. 2006 Oct 116(10): 1882-6.

13. Schlosser RJ, Woodworth BA, Wilensky EM, Grady MS, Bolger WE. Spontaneous cerebrospinal fluid leaks: a variant of benign intracranial hypertension. Annals of Otology, Rhinology \& Laryngology. 2006 Jul;115(7):495-500. 